\title{
Transient Abnormal Myelopoiesis Associated with Down Syndrome
}

National Cancer Institute

\section{Source}

National Cancer Institute. Transient Abnormal Myelopoiesis Associated with Down

Syndrome. NCl Thesaurus. Code C82339.

A myeloid proliferation occurring in newborns with Down syndrome. It is clinically and morphologically indistinguishable from acute myeloid leukemia and is associated with GATA1 mutations. The blasts display morphologic and immunophenotypic features of megakaryocytic lineage. In the majority of patients the myeloid proliferation undergoes spontaneous remission. 